\title{
Relación entre laAgilidad respecto de Variables Antropométricas en niños pertenecientes a una Escuela de tenis privada de la provincia de Concepción \\ Relationship between Agility and Anthropometric Variables in children from a private tennis school in the province of Concepción \\ *; **Pablo Luna Villouta; ***Cristian Márquez Sánchez; **Marcelo Rodriguez Gallardo; ****Cristian Martínez Salazar **Rodrigo Vargas Vitoria \\ *Universidad San Sebastián (Chile); **Universidad Católica del Maule Chile); ***Andrés Bello, Concepción (Chile); ****Universidad de La Frontera (Chile)
}

Resumen. El tenis es un tipo de deporte caracterizado por esfuerzos cortos e intermitentes, la evidencia apoya el argumento de que las capacidades funcionales como la fuerza, la potencia, la velocidad y la agilidad son necesarias para competir en los niveles más altos. La investigación buscó analizar la relación entre la agilidad y las variables antropométricas (peso y talla) en niños pertenecientes a una escuela de tenis privada de la provincia de Concepción. El estudio utilizó un enfoque cuantitativo, de carácter descriptivo correlacional, de corte transversal. La muestra de 28 niños, de entre ocho y 11 años de edad, fue seleccionada de forma no probabilística por conveniencia. Se evaluó el peso y la estatura como variables antropométricas; para evaluar la agilidad se utilizó el Test MAT (Modified Agility Test). Los resultados mostraron que el tiempo promedio obtenido para la Agilidad fue de 9,10 $\pm 1,01$ segundos y que existe una baja relación entre la agilidad y las variables antropométricas de peso $(r=-.189 ; p=.336)$ y talla $(r=-.176 ; p=.391)$. Se concluye que la agilidad presenta una baja relación con las variables antropométricas estudiadas.

Palabras clave: Agilidad, tenis, niños, peso, talla.

Abstract. Tennis is a type of sport characterized by short and intermittent efforts. Evidence supports the argument that functional abilities such as strength, power, speed, and agility are necessary to compete at the highest levels. This research sought to analyze the relationship between agility and anthropometric variables (weight and height) in children belonging to a private tennis school in the province of Concepción. The study used a quantitative, descriptive, correlational, cross-sectional approach. A sample of 28 children between eight and 11 years of age was selected employing a non-probabilistic sampling technique by convenience. Weight and height were evaluated as anthropometric variables; The MAT Test (Modified Agility Test) was used to evaluate Agility. Results showed that average time obtained for agility was $9.10 \pm 1.01$ seconds, showing a moderate correlation with the anthropometric variables of weight $(r=, 189 ; p=, 336)$ and height $(r=-, 176 ; p=, 391)$. In conclusion, agility has a low correlation with the anthropometric variables studied.

Key-works: Agility, tennis, children, Weight, Size

\section{Introducción}

El tenis es un tipo de deporte caracterizado por esfuerzos cortos e intermitentes de una intensidad alta y con tiempos de juego cambiantes, donde numerosos factores determinan el éxito alcanzado. Algunos de ellos incluyen la constitución corporal morfológica del ser humano, las habilidades motoras y la eficiencia de los sistemas energéticos (Karnia, Garsztka, Zurek, Luszczyk, Eledziewska \& Ziemman, 2010).

El esfuerzo físico durante los partidos de tenis fluctúa entre períodos cortos de intensidad máxima y períodos más largos de intensidad moderada a baja (Fernandez, 2006). Los puntos tienen una duración aproximada de 8 o 10 segundos de juego, con 20 segundos de descanso, durante los puntos existe en promedio un intercambio de 10 golpes con cuatro cambios de dirección, la mayoría de los sprint se realizan en distancias comprendidas entre 2,5 y 6 metros.

En los últimos años, el tenis ha cambiado, en primer lugar, el juego se ha vuelto más rápido, donde ocurren varios cambios de dirección. En segundo lugar, hoy en día involucra a jugadores de menor edad, que se entrenan con alta intensidad y carga de trabajo. Esto indica la necesidad de un análisis y una evaluación más profundos de la capacidad física y las capacidades motoras en cada etapa del desarrollo del

Fecha recepción: 29-10-18. Fecha de aceptación: 15-03-19 Rodrigo Vargas Vitoria

rvargas@ucm.cl jugador (Karnia, et al. 2010).

Dadas las condiciones generales anteriores, se requiere una combinación de capacidades funcionales de los jugadores, aunque la combinación exacta de capacidades aún no se ha determinado (Kovacs, 2007). Sin embargo, la evidencia apoya el argumento de que las capacidades funcionales como la fuerza, la potencia, la velocidad, la agilidad y la resistencia son necesarias para competir en los niveles más altos (Myburgh, Cumming, Silva, Cooke, \& Malina, 2016).

La agilidad es un importante y necesario componente físico en las actividades deportivas y recreativas (Pauole, Madole, Garhammer, Lacourse, \& Rozenek, 2000). Tradicionalmente, es definida como la capacidad o habilidad de cambiar de modo rápido y seguro una conducta espacial, la dirección de un movimiento o el sentido de un desplazamiento (Martinez, 2002).

De acuerdo a autores, tales como Sassi, Dardouri, Yahmed, Gmada, Mahfoudhi, \& Gharbi (2009);; Draper \& Lancaster (1985), el término agilidad permite describir una acción dinámica, donde se producen cambios en la posición corporal y modificaciones de la dirección de la carrera; de esta forma, la agilidad, puede ser clasificada como una habilidad perceptivo-motriz, fundamental para el tenis, ya que permite al jugador colocarse en posición correcta y sólida desde la cual golpear la pelota y dirigir su juego (Crespo \& Miley, 1999).

Estudios recientes afirman que la agilidad, está determinada por diversos factores, no requiere únicamente de cam- 
bios de dirección a alta velocidad, sino que también son necesarios aspectos perceptuales (Sassi et al. 2009), los que permiten al sujeto dirigir y controlar sus movimientos a una alta velocidad y con máxima precisión, todo en un espacio y en tiempo determinado.

El posicionamiento del cuerpo y de los pies, los movimientos hacia la pelota, la colocación alrededor de ella, la posición de golpeo y la recuperación de la posición son fundamentales en el tenis actual, para cumplir con el plan de juego y lograr buenos resultados. Así, se ha visto que la agilidad juega un papel determinante para el rendimiento, ya que el jugador de tenis debe estar cambiando de posición constantemente y, casi siempre, a una gran velocidad, en diferentes direcciones y con diversos movimientos (desplazamientos laterales, frontales, saltos, giros, etc. (Sekulic, Uljevic, Peric, Spasic, \& Kondric, 2017).

Por lo anterior, está siendo aceptado actualmente que la agilidad es una importante medida de calidad en la mayoría de los deportes donde un rápido y efectivo cambio planificado o no planificado de dirección es obligatorio (Sattler, Sekulic, Spasic, Peric, Krolo, Uljevic \& Kondric, 2015; SánchezMuñoz, Sanz, \& Zabala 2007).

Así, la agilidad es una cualidad muy importante en el tenis, sin embargo, hay una evidente falta de estudios que se centren en la aplicabilidad de las actuaciones de agilidad específicas en el tenis (Sekulic, et al. 2017), recientemente, ha habido un creciente interés en los representantes del tenis juvenil en conocer los factores que influyen en su rendimiento, pero solo algunos datos ya han sido publicados, por lo que el tema aún está abierto, más aún en la relación entre el crecimiento corporal, la capacidad física o las habilidades técnicas específicas de los jugadores jóvenes (Ziemann, Eledziewska, Grzywacz, Gibson \& Wierzba, 2011), es así como en varias ocasiones se intenta explicar que la capacidad de los tenistas de moverse ágilmente por la cancha se debe a la talla, el peso o la composición corporal, lo que no está determinado, requiriéndose mayores evidencias al respecto (Karnia, et al. 2010; Kovacs, 2007).

Considerando lo anterior, es necesario evaluar las relaciones entre los distintos factores que pueden determinar el nivel de las principales cualidades físicas para el éxito deportivo, tal como lo son las variables antropométricas en cualquier especialidad deportiva (Baiget, Rodríguez \& Iglesias, 2016), para así favorecer el adecuado diseño del proceso de entrenamiento en sus distintas etapas.

A partir de los antecedentes mencionados, se plantea el objetivo de analizar la relación entre la agilidad y las variables antropométricas de peso y talla en niños pertenecientes a una Escuela de tenis privada de la provincia de Concepción.

\section{Método}

Estudio Descriptivo correlacional de corte transversal. A través de un muestreo no probabilístico por conveniencia, se seleccionó a 28 sujetos que practican tenis de entre ocho años seis meses y 11 años, 9 meses, pertenecientes a una Escuela de tenis privada de la provincia de Concepción. La institución deportiva fue seleccionada por su disponibilidad de participación, además se ponderó que contaran con acti- vidades deportivas planificadas y ejecutadas sistemáticamente (cuatro veces por semana), con gimnasio techado (lo que reduce las suspensiones debido a las lluvias y frio de invierno) y a cargo de personal técnico certificado por la Federación de Tenis de Chile (FETECH) o la Federación Internacional de Tenis (ITF).

Los criterios de Inclusión para los participantes fueron: 1) Pertenecer a la Escuela de tenis privada seleccionada; 2) Tener entre ocho y 11,9 años de edad al momento de las evaluaciones; 2) a la fecha de las evaluaciones haber asistido regularmente a las clases de tenis, $75 \%$ de asistencia., durante los últimos 12 meses; 4) firma del consentimiento informado por parte del apoderado. Por su parte, se consideraron como criterio de exclusión, 1) no completar las evaluaciones. 2) no asistir a las sesiones programadas de familiarización con el test 3) sufrir algún tipo de lesión o discapacidad física u otra, que afecte el resultado de las evaluaciones.

Como procedimiento se seleccionó a escuelas de tenis privadas de Concepción, que contaran con clases de tenis ejecutadas sistemáticamente, con gimnasio techado y a cargo de personal técnico certificado por la Federación de Tenis de Chile (FETECH) o la Federación Internacional de Tenis (ITF). Se les envió una carta a los directivos declarando el objetivo de la investigación e invitándolos a participar. Luego de esto, se les envió un consentimiento informado a los apoderados de los sujetos, con el fin de informar respecto del objetivo del estudio, el carácter anónimo y voluntario de la participación, y que las evaluaciones no revestían ningún perjuicio para la salud de los menores; el consentimiento debía ser firmado por los apoderados autorizando la participación de su pupilo, luego este firmó el asentimiento, ratificando su participación en las evaluaciones. El estudio contó con la aprobación del comité de ética de la Universidad San Sebastián, Chile, por lo que los procedimientos se realizaron bajo los principios establecidos en la declaración de Helsinki (Asociación Médica Mundial, 2014).

La asistencia regular a las clases de Tenis de los menores fue proporcionada por la coordinación deportiva del club privado. Las variables antropométricas se evaluaron descalzos, con pantalón corto y camiseta. La masa corporal (kg) se evaluó utilizando una balanza mecánica (Seca 700), con una precisión de 50 grs, que oscila entre 0 a $220 \mathrm{~kg}$, la talla (cm), se midió según el plano de Frankfurt sin zapatos, utilizando un estadiómetro de aluminio de marca Seca 220, graduada en milímetros, su escala fue de $(60-220$ cm). Para la evaluación de la agilidad, se utilizó el test MAT (Modified Agility Test) propuesto por Sassi, et al. (2009). Para el Test MAT (Figura $1)$, cuatro conos eran dispuestos en forma de «T», con el cono B colocado $5 \mathrm{~m}$ del cono A, y dos conos adicionales (C y D) colocados a 2,5 m a los lados del cono B. Se les pidió a los sujetos que realizaran el siguiente recorrido:

- Cono A al B: Desplazamiento hacia delante hasta tocar el cono B con la mano derecha.

- Cono B-C: Desplazamiento lateral a la izquierda, sin superar en ningún momento la línea de conos, hasta tocar el cono $C$ con su mano izquierda.

- Cono C-D: Desplazamiento lateral a la derecha hasta tocar el cono D con la mano derecha.

- Cono D-B: Desplazamiento lateral a la izquierda 
hasta tocar el cono B con la mano izquierda.

- Cono B-A: Desplazamiento hacia atrás al punto de partida A con una carrera de espaldas.

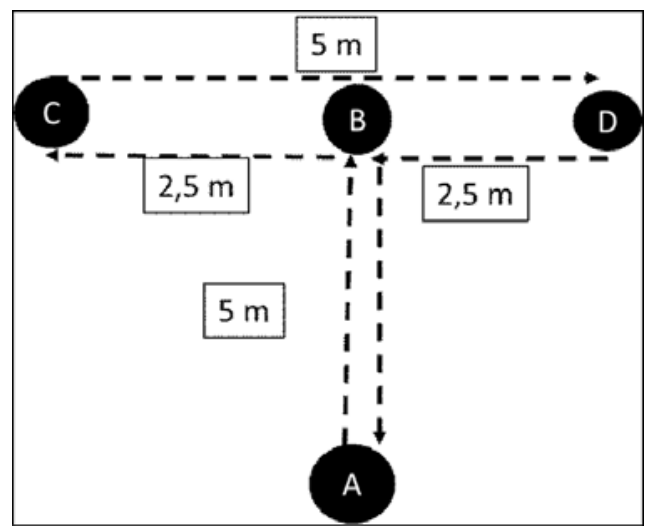

Figura 1. Recorrido utilizado en Test MAT

La distancia total en cada serie fue de $20 \mathrm{~m}$.

Los desplazamientos laterales se debían realizar sin cruzar las piernas.

Se realizaron 3 repeticiones del recorrido descrito en el menor tiempo posible, con un descanso de 4 a 5 minutos entre cada ejecución. La salida desde el punto inicial, partiendo desde una posición retrasada $0,5 \mathrm{~m}$ respecto al cono A, se realizaba cuando el ejecutante lo considerase oportuno. La posición de salida fue en bipedestación con una pierna adelantada sobre la otra.

La recolección de datos se durante agosto del año 2017. Todo el procedimiento de evaluación, fue realizado en los en las canchas de arcilla del club, en horario de clases y estuvo a cargo de evaluadores que poseían la experiencia necesaria para la recolección de la información, y que además fueron capacitados para la toma de las pruebas. Adicionalmente, se realizaron 2 sesiones de familiarización previa de los participantes en las que recibieron instrucciones y explicaciones por parte de los investigadores de la correcta realización del test. Todos los evaluados pudieron experimentar la ejecución del test, realizándolo entre 6 y 8 ocasiones, en sesiones de la semana previa a la realización de las evaluaciones.

\section{Resultados}

A continuación, se presentan los resultados de la muestra evaluada, se verificó la normalidad de los datos del Test MAT (Modified Agility Test) por medio de la prueba de Shapiro- Wilk ( $p=0.510)$. Se efectuó un análisis estadístico descriptivo, para caracterizar a la muestra, utilizando medidas de tendencia central como la media aritmética, además de medidas de variabilidad como puntaje mínimo, puntaje máximo y desviación estándar. Para probar que existe una relación significativa entre las variables antropométricas, se utilizó una prueba de hipótesis asociada al coeficiente de

Tabla 1

Resumen Exploratorio para las variables antropométricas y de agilidad que caracterizan a la muestra del estudio:

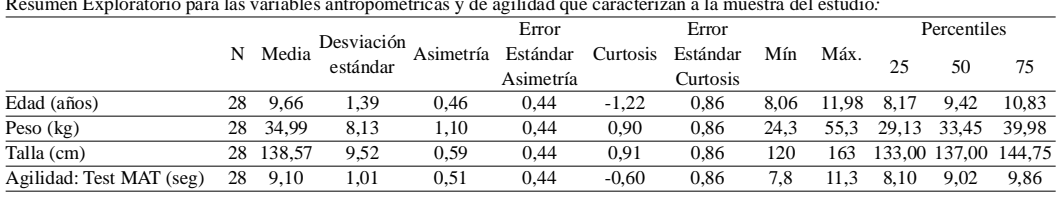

Pearson, el nivel de significancia adoptado fue de $p<0.05$. Los cálculos se efectuaron con planillas de Excel 2016 y con el software estadístico SPSS 20.0.

La Tabla 1, presenta un resumen exploratorio de los datos, donde se observa una agilidad promedio de 9,10 segundos y una desviación con respecto a la media de 1,01 segundos. Además, el grupo es homogéneo ya que el 25\% de los individuos tiene una agilidad inferior a 8,17 segundos y el $25 \%$ superior de los individuos tiene una agilidad superior a 9,86 segundos. Con respecto a la asimetría, para la agilidad se observa una asimetría muy leve (0.51), característico de datos con distribución normal. Por otro lado, la edad se concentra entre los ocho y 11,9 años, el peso entre los veinticuatro y $55 \mathrm{~kg}$. y la talla entre ciento veinte y $163 \mathrm{~cm}$.

En la Tabla 2, se observa una relación moderada en inversa entre la Agilidad y las variables antropométricas de peso $(r=-0.189 ; \mathrm{p}=0.336)$ y talla $(r=-0.176 ; p=0.391)$ De esta forma, la Agilidad no está determinada por las variables antropométricas evaluadas.

En la Figura 2, se observa una débil relación entre la agilidad y las variables peso y talla. La afirmación anterior es concordante con la Tabla 2 donde la significancia bilateral es superior al $5 \%$.

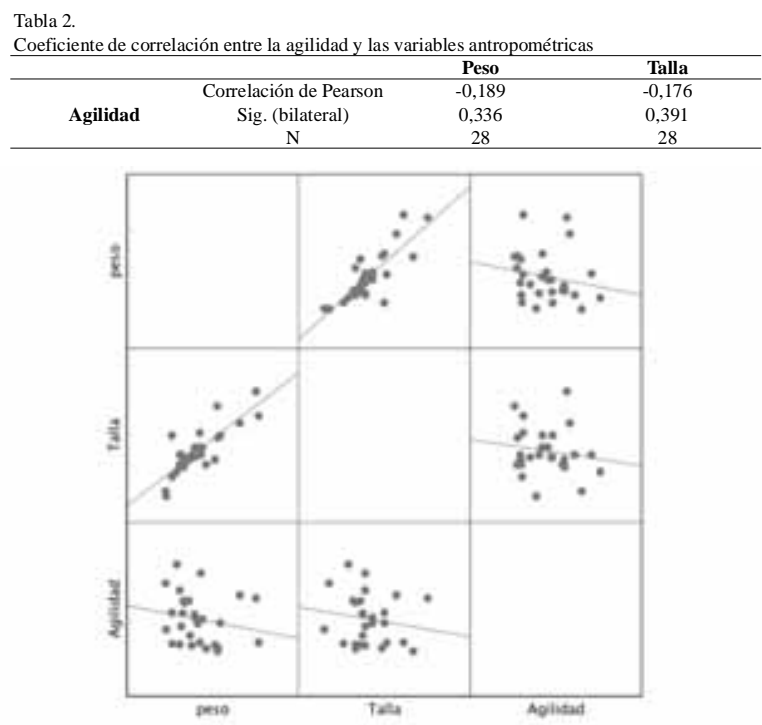

Figura 2. Diagrama de dispersión entre agilidad, peso y talla

\section{Discusión}

En relación a los resultados obtenidos, al comparar el promedio de tiempo alcanzado por los evaluados en el test de agilidad, 9,10 $\pm 1,01$ segundos con escolares españoles testeados por Yanci, Los Arcos, Reina, Gil, \& Grande (2014), que utilizaron el mismo test que el presente estudio, se observa que los menores que practican tenis fueron más rápidos que los escolares españoles, quienes completaron el recorrido en mayor tiempo (Media= 9,76 $\pm 0,90$ segundos), estos resultados son coincidentes con otras indagaciones en población chilena que han detectado que recibir estimulación motriz más allá de las clases escolares sistemáticas de Educación Física, es beneficio suficiente para lograr mejores resultados y soslayar los 
déficits motrices del creciente estilo de vida sedentario de la población en general (Pradenas, Campos, Contreras, Puentes, \& Luna, 2017; Luna, Aravena, Contreras, Fabres, \& Faúndez, 2016; Leiva, Alvarado, Gallardo, Vargas, \& Martínez, 2015; Fritz \& Vargas, 2013).

Lo anterior, coincide con los aportes de otros autores (Farrow, Young \& Bruce, 2005; Keogh, Weber \& Dalton 2003; Shala, 2009) quienes destacan que los sujetos que reciben una adecuada orientación de actividades perceptivomotrices, con metodologías especificas e innovadoras, logran obtener mejores resultados al mostrarse mejor capacitados que quienes no practican o lo hacen inadecuadamente.

De la misma forma, Yanci et al. (2014), plantean que quizá sea interesante incluir programas específicos de agilidad en las sesiones de educación física, dado su carácter multilateral. Lo anterior, debe propiciar la reflexión en el sentido de considerar la diversificación de actividades motrices y la influencia de factores externos en el desarrollo de las habilidades perceptivo motrices, como la agilidad, tales como la cantidad y la calidad de la estimulación motriz, la orientación metodológica y didáctica de la iniciación deportiva durante la niñez.

Los resultados derivados para la agilidad, por medio del Test Mat, permiten observar que los requerimientos específicos del tenis, tales como el posicionamiento del cuerpo y de los pies, los movimientos rápidos y explosivos hacia la pelota, la colocación alrededor de la pelota, la posición de golpeo y la recuperación de la posición durante los juegos y ejercitaciones de tenis, son una adecuada estimulación para el desarrollo de la agilidad. Esto refuerza su rol dentro del tenis, lo que está en línea a lo descrito por (Sekulic, et al. 2017) quienes destacan que la agilidad juega un papel determinante para el control del juego y del rendimiento, ya que el jugador de tenis debe estar cambiando de posición constantemente y casi siempre a una gran velocidad, en diferentes direcciones y con diversos movimientos (desplazamientos laterales, frontales, saltos, giros, etc.)

Por su parte, al relacionar la agilidad con las variables antropométricas estudiadas, peso y talla, se encontró una relación baja con ambas variables, peso $(r=-, 189 ; p=, 336)$ y talla $(r=-, 176 ; p=, 391)$, lo que significa que la agilidad en este grupo de menores que practican regularmente tenis, no está determinada fundamentalmente por estas variables antropométricas. Estos resultados son coincidentes con lo reportado por Caballero-Ruíz, Carrasco-Legleu, De León, Candia-Luján \& Ortiz-Rodríguez (2019), quienes señalan que las características corporales por si solas no parecen ser un determinante del rendimiento deportivo, debiéndose supervisar la interacción de estas con otras valencias físicas y fisiológicas, lo que permitirá orientar adecuadamente las propuestas de entrenamiento.

Lo anterior, viene a demostrar que el rendimiento y la selección del talento deportivo, son multifactoriales, debiéndose considerar que las características físicas y morfológicas son variables en función de la modalidad deportiva, más aún de acuerdo al entrenamiento, la táctica y la técnica elegida por el deportista y equipo técnico, estos aspectos son parte de la discusión actual en las ciencias del deporte (Borges, Ruiz \& Argudo, 2017; Zuñiga, Osorio, de Toledo \& Herrera, 2018). Por lo tanto, no se debe presuponer que el resultado de la competencia estará determinado por ciertas características corporales del tenista, ya que existe poca información sobre la interacción de los distintos parámetros corporales y físicos determinantes del rendimiento, más aún en edades formativas iniciales.

Junto con ello, este hallazgo reafirma los planteamientos actuales acerca de que la agilidad, como una cualidad no depende prioritariamente de las características corporales del deportista (Sassi, et al. 2009, Crespo \& Miley, 1999; Sattler, et al. 2015; Sánchez-Muñoz, Sanz \& Zabala, 2007) sino que la ubican como una compleja habilidad perceptivo motriz, que obedece a variados factores, tanto físicos como motores enfatizando en los aspectos de control corporal y perceptuales de espacio-tiempo, como fundamentales para controlar y cambiar rápidamente los movimientos con alta precisión para así prevalecer en el juego.

Teniendo en cuenta lo anterior, la agilidad con una correcta estimulación a cargo del personal técnico deportivo debidamente capacitado, puede presentar buenos valores, más allá de las características corporales como el peso y la talla, por lo que estos resultados pueden servir de referencia para la planificación del entrenamiento y para otros estudios nacionales, que consideramos necesarios de elaborar para aumentar el incipiente conocimiento científico asociado a la formación deportiva inicial.

Por el contrario, como limitaciones es necesario señalar que el grupo evaluado presenta similares características corporales, por lo que los resultados obtenidos y la relación entre las valencias evaluadas, pueden variar en otras poblaciones de diferentes características. Además, lo reducido de la muestra evaluada $(\mathrm{n}=28)$, su selección no-probabilística y la interacción con otras cualidades físicas, son otras limitaciones que también se sugiere controlar, puesto que aquello podría aportar información relevante y enriquecer la información de futuros estudios.

\section{Conclusión}

Al analizar la relación entre la agilidad y las variables antropométricas de peso y talla en niños pertenecientes a una Escuela de tenis privada de la provincia de Concepción, los resultados muestran que no existe una relación significativa entre la agilidad y las variables antropométricas. De igual forma, la agilidad se presentó en adecuados valores para la muestra evaluada, lo que sugiere que el tenis parece ser un deporte que la estimula de manera apropiada, y que la agilidad es una compleja habilidad perceptivo-motriz, que depende de factores de control corporal y perceptuales de espacio-tiempo, más allá de las características corporales del deportista.

\section{Referencias}

Asociación Médica Mundial. (2014). Declaración de Helsinki. Principios éticos para la investigación en seres humanos. Boletín del Consejo Académico de Ética en Medicina. 1(2): 239-243.

Baiget, E., Rodríguez, F.A. e Iglesias, X. (2016) Relación entre parámetros técnicos y fisiológicos en tenistas de compe- 
tición. Revista Internacional de Medicina y Ciencias de la Actividad Física y el Deporte, 16 (62), 243-255. http:/ /dx.doi.org/10.15366/rimcafd2016.62.005

Borges Hernández, P., Ruiz Lara E. \& Argudo Iturriaga F. (2017). Relación entre parámetros antropométricos, agarre máximo y velocidad de lanzamiento en jugadores jóvenes de waterpolo. Retos. Nuevas tendencias en Educación Física, Deporte y Recreación, (31), 212-218.

Caballero-Ruíz A., Carrasco-Legleu C., De León L., CandiaLuján R. \& Ortiz-Rodríguez B. (2019). Somatotipo de mujeres futbolistas universitarias por posición en el terreno de juego. Retos. Nuevas tendencias en Educación Física, Deporte y Recreación, (36), 228-230.

Crespo, M., \& Miley, D. (1999). Manual de Entrenadores Avanzados. Londres: ITF Ltd.

Draper JA. \& Lancaster MG. (1985). The 505 test: A test for agility in the horizontal plane. Aust J. Sci Med Sport, 17, 15-18.

Farrow, D. Young, W. \& Bruce, L. (2005). The development of a test of reactive agility for netball: a new methodology. Journal of Science and Medicine in Sport, 8(1), 52-60. doi:10.1016/s1440-2440(05)80024-6

Fernandez, J. (2006). Intensity of tennis match play. British Journal of Sports Medicine, 40(5), 387-391. doi:10.1136/ bjsm.2005.023168

Fritz, J. \& Vargas, R. (2013). Impacto de actividades lúdicas no competitivas sobre las habilidades motoras gruesas en niños y niñas pre escolares. Revista Ciencias de la Actividad Física UCM, 14(1), 31-37

Karnia M. Garsztka T., - urek P., £uszczyk M., Eledziewska E. \& Ziemann E. (2010) Body composition and physical capacity of elite adolescent female tennis players. Georgian medical news, 19(27), 113-123.

Keogh, J, Weber, CL. \& Dalton, CT. (2003). Evaluation of anthropometric, physiological, and skill-related test for talent identification in female field hockey. Can J Appl Phys, 28, 397-409.

Kovacs MS. (2007) Tennis physiology. Training the competitive athlete. Sports Medicine, 37(3),189-198.

Leiva, M., Alvarado, C., Gallardo, R., Vargas, R., \& Martínez, C. (2015). Desarrollo motor en escolares con diferentes aprestos formativos motrices. Revista Ciencias de la Actividad Física UCM, 16(1), 19-28.

Luna, P., Aravena, J., Contreras, D., Fabres, C., \& Faúndez, F. (2016). Efectos en el desarrollo motor de un programa de estimulación de habilidades motrices básicas en escolares de $5^{\circ}$ año Básico de colegios particulares subvencionados de Concepción. Revista Ciencias de la Actividad Física UCM,17(1), 27-36.

Martinez López, E. (2002). Pruebas de Aptitud Física. Barcelona: Paidotribo.

Myburgh, G. K., Cumming, S. P., Silva, M. C. E., Cooke, K., \& Malina, R. M. (2016). Maturity-Associated Variation in Functional Characteristics Of Elite Youth Tennis Players. Pediatric Exercise Science, 28(4), 542-552. doi:10.1123/ pes.2016-0035

Pauole, K, Madole, K, Garhammer, J, Lacourse, M, \& Rozenek, R. (2000). Reliability and validity of T-Test as a measure of agility, leg power, and leg speed in college-aged men and women. J Strength Cond Res, 14(4), 443-450.
Pradenas, X.; Campos, M.; Contreras, M.; Puentes,D. \& Luna, P. (2017). Comparación del desarrollo motor en escolares de 9 y 10 años de edad en clases de educación física y talleres deportivos extracurriculares. Revista Ciencias de la Actividad Física UCM, 18(2), 1-8. doi.org/ 10.29035/rcaf.18.2.1

Sanchez-Muñoz, C., Sanz, D., \& Zabala, M. (2007). Anthropometric characteristics, body composition and somatotype of elite junior tennis players. British Journal of Sports Medicine, 41(11), 793-799. doi:10.1136/ bjsm.2007.037119

Sassi, RH, Dardouri, W, Yahmed, MH, Gmada, N, Mahfoudhi, ME, \& Gharbi, Z. (2009). Relative and absolute reliability of a Modified Agility T-Test and its relationship with vertical jump and straight sprint. J Strength Cond Res, 23(6), 1644-1651.

Sattler, T., Sekulic, D., Spasic, M., Peric M., Krolo, A., Uljevic, O. \& Kondric, M. (2015). Analysis of the Association Between Motor and Anthropometric Variables with Change of Direction Speed and Reactive Agility. Performance. J Hum Kinet, 47(1), 137-145. doi:10.1515/hukin2015-0069

Sekulic, D., Uljevic, O., Peric, M., Spasic, M., \& Kondric, M. (2017). Reliability and Factorial Validity of Non-Specific and Tennis-Specific Pre-Planned Agility Tests; Preliminary Analysis. Journal of Human Kinetics, 55(1), 107-116. doi:10.1515/hukin-2017-0010

Shala, M. (2009). Assessing gross motor skills of Kosovar preschool children. Early Child Development and Care, 179(7), 969-976. doi:10.1080/03004430701667452

Yanci, J., Los Arcos, A., Reina, R., Gil, E. \& Grande, I. (2014). La agilidad en alumnos de educación primaria: diferencias por edad y sexo. Revista Internacional de Medicina y Ciencias de la Actividad Física y el Deporte, 14(53), 23-35.

ZiemannE., EledziewskaE., Grzywacz T., GibsonA. \& Wierzba, T. (2011). Body composition and physical capacity of elite adolescent female tennis players. Georgian Medical News, 7(196), 19-27.

Zuñiga U., Osorio A., Toledo. I. \& Herrera, R. (2018). Somatotipo en futbolistas mexicanos profesionales de diferente nivel competitivo. Retos. Nuevas tendencias en Educación Física, Deporte y Recreación, (34), 100102.

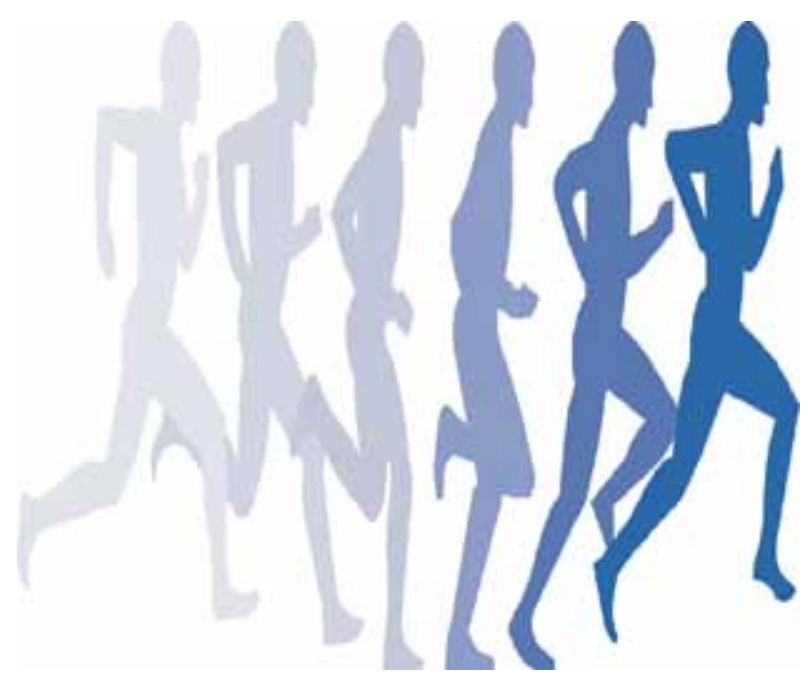

